\title{
Gynecological/Obstetrical Nursing
}

National Cancer Institute

\section{Source}

National Cancer Institute. Gynecological/Obstetrical Nursing. NCI Thesaurus. Code

C18827.

Nursing care of women, specifically with regard to childbearing and gynecological care. 